\title{
INTERCORRÊNCIAS OBSTÉTRICAS QUE OCASIONAM O PRÉ-NATAL DE ALTO RISCO
}

\author{
OBSTETRIC INTERCURRENCES THAT CAUSE HIGH RISK PRE \\ CHRISTMAS
}

\author{
Maria Eduarda Otoni Da Silva Garrido ${ }^{1}$ \\ Anne Caroline de Souza ${ }^{2}$ \\ Roneiza Soares Rufino ${ }^{3}$ \\ Teógenes de Oliveira ${ }^{4}$ \\ Ocilma Barros de Quental ${ }^{5}$ \\ Macerlane de Lira Silva ${ }^{6}$
}

RESUMO: a assistência ao pré-natal é a melhor forma da prevenção de complicações durante o período gestacional e o parto, sendo assim, para um melhor resultado, as consultas devem ser realizadas por uma equipe multiprofissional capacitada, capaz de reconhecer as necessidades das gestantes durante toda a gravidez até o período puerperal. Este estudo foi realizado em 2021, e trata-se de uma revisão integrativa da literatura, que foi desenvolvida nas bases de dados Scientific Electronic Library Online (SCIELO), Biblioteca Virtual de Saúde (BVS) e sites do Ministério da Saúde, utilizando os descritores: "Gravidez", "Assistência prénatal", "Gestação de alto risco", "Complicações na gravidez". Pesquisaram-se documentos dos últimos cinco anos, na íntegra, gratuitos e que respondessem a questão norteadora inicial. No ano de 2000 , a mortalidade materna era um dos principais problemas mundiais, e, após implementação de programas e políticas de saúde às gestantes e puérperas, houve uma significativa melhoria na assistência materno-infantil, resultando na diminuição da mortalidade, porém, os números de intercorrências ainda são considerados altos. As principais causas de intercorrências são os fatores adquiridos durante a gestação, que evolui como risco gestacional,

\footnotetext{
1 Enfermeira. Faculdade Santa Maria de Cajazeiras. eduardaotoni89@gmail.com.

2 Enfermeira. Especialista em docência do ensino superior. Docente da Faculdade Santa Maria. annekarolynne20@hotmail.com.

${ }^{3}$ Enfermeira. Faculdade Santa Maria de Cajazeiras. Roneiza.soares30@gmail.com.

4 Médico. Residente em Medicina de Família e Comunidade. Fauldade Santa Maria - FSM. teogenesoliveira@gmail.com.

5 Enfermeira. Doutora em ciências da Saúde. Docente da Faculdade Santa Maria - FSM. Dra.quental@gmail.com.

6 Enfermeiro. Mestre em Saúde Coletiva. Docente da Faculdade Santa Maria - FSM. marcerlane@hotmail.com.
} 
como, por exemplo, hemorragias, pré-eclâmpsia ou eclampsia, diabetes gestacional, quantidade de fetos e de líquido amniótico, contração de doenças infectocontagiosas, exposição a agentes teratogênicos ou óbito. Sendo assim, é importante investigar lacunas na atenção primária do pré-natal, fortalecer a atenção especializada e manter as políticas públicas, com vistas a otimizar os indicadores de saúde materna e infantil.

Descritores: Gravidez. Assistência pré-natal. Gestação de alto risco. Complicações na gravidez.

ABSTRACT: prenatal care is the best way to prevent complications during the gestational period and childbirth, so, for a better result, consultations must be carried out by a trained multiprofessional team, capable of recognizing the needs of pregnant women throughout the entire period. Pregnancy to the puerperal period. This study was carried out in 2021 and is an integrative literature review, which was developed in the Scientific Electronic Library Online (SCIELO), Virtual Health Library (BVS) and Ministry of Health websites, using the descriptors: "Pregnancy", "Prenatal care", "High-risk pregnancy", "Pregnancy complications". Documents from the last five years were searched, in full, free of charge and that answered the initial guiding question. In the year 2000, maternal mortality was one of the main global problems and after the implementation of health programs and policies for pregnant and postpartum women, there was a significant improvement in maternal and child care, resulting in a decrease in mortality, however, the numbers of complications are still considered high. The main causes of complications are the factors acquired during pregnancy that evolve as a risk of pregnancy, such as bleeding, preeclampsia or eclampsia, gestational diabetes, amount of fetuses and amniotic fluid, contracting infectious diseases, exposure to teratogenic agents or death. Therefore, it is important to investigate gaps in primary prenatal care, strengthen specialized care and maintain public policies with a view to optimizing maternal and child health indicators.

Descriptors: Pregnancy. Prenatal care. High-risk pregnancy. Pregnancy complications. 


\section{INTRODUÇÃO}

A gestação é um período que envolve mudanças físicas, psicológicas e sociais. Durante o curso da gestação, a mulher está susceptível a diversas variáveis que podem levar a um prognóstico materno e/ou fetal desfavorável, determinando uma gestação de alto risco, por exemplo, a elevação pressórica é uma das principais alterações que culminam com uma gestação de risco aumentado (SAMPAIO; ROCHA; LEAL, 2018).

O acompanhamento pré-natal tem por objetivo garantir que a gestação prossiga sem intercorrências. Os primeiros passos para um pré-natal de qualidade são o acolhimento e a escuta qualificada, de forma ética e sem julgamentos ou preconceitos, com garantia à privacidade e confidencialidade no que for dito, isso fortalecerá a relação de confiança, comprometimento e vínculo entre o profissional e a gestante. É também no acolhimento onde será definida a prioridade clínica da gestante, através da história clínica, avaliação física e complementar, dando continuidade durante todas as consultas pré-natal (BRASIL, 2016).

A gestação é considerada de alto risco quando a saúde ou a vida da mãe e/ou do feto, ou do recém-nascido, podem ser afetados, requerendo maior vigilância para prevenir o surgimento de algum fator de risco, ou, em alguns casos, os fatores de risco já podem estar presentes antes mesmo da concepção, dessa forma, tornase essencial que todo profissional seja capaz de avaliar e identificar o momento em que essa mulher irá precisar de um cuidado mais ampliado e vigilante (BRASIL, 2012; GADELHA et al., 2020).

No Brasil, a prevalência da diabetes gestacional e a hipertensão gestacional variam de 0,8 a $34,5 \%$, essas são as principais causas de morbimortalidade materna e perinatal do mundo, porém existem outros fatores, como as condições clínicas obstétricas associadas a outras complicações, condições sociodemográficas, história reprodutiva, obesidade. A presença de um ou mais desses fatores não significa que a gestante necessita de cuidados imediatos com tecnologia avançada, mas que 
essa mulher precisa de uma atenção maior, ações educativas e um intervalo menor entre as consultas, sendo assim, a propedêutica vai variar a depender das condições da gestante e da causa desse risco (SAMPAIO; ROCHA; LEAL, 2018).

As síndromes hipertensivas constituem a maior causa de mortalidade materna e perinatal no Brasil, principalmente quando associadas as suas complicações, como eclâmpsia e síndrome de HELLP, além disso, outras intercorrências de grande frequência são: diabetes mellitus gestacional, oligodramnia e amniorrexe prematura; e as complicações obstétricas mais relatadas foram: parto prematuro, hemorragias no pós-parto, infecções das vias urinarias, sofrimento fetal e crescimento intrauterino restrito (SOARES; BORGES; BORGES, 2016).

Diante do que foi exposto, e levando em consideração a importância do prénatal, é fundamental que se busque as causas da gestação de alto risco e, assim, se possível, prevenir e controlar as complicações gestacionais. Dessa forma, a pesquisa objetiva entender as causas da gravidez de alto risco e as intercorrências presenciadas durante o pré-natal.

\section{MÉTODO}

Trata-se de um estudo não-observacional, descritivo, do tipo revisão integrativa da literatura, como método importante na análise de pesquisas que dão suporte para a tomada de decisão e a melhoria da prática clínica. A escolha da revisão integrativa como método de pesquisa é justificada por ser um método que favorece incorporar evidências científicas atualizadas na prática, uma vez que, a compilação de informações em meios eletrônicos é um grande avanço para os pesquisadores, democratizando o acesso e proporcionando atualização constante (MENDES; SILVEIRA; GALVÃO, 2008). A pesquisa foi desenvolvida em seis etapas: 1) delimitação do tema; 2) critérios de inclusão e exclusão; 3) coleta de dados; 4) avaliação dos estudos; 5) discussão e interpretação dos resultados; 6) apresentar os resultados da pesquisa. 
O estudo tem o foco de responder a pergunta norteadora: quais são as intercorrências e complicações que podem desenvolver uma gestação de alto risco? A busca foi realizada nas bases de dados Scientific Electronic Library Online (SCIELO), Biblioteca Virtual de Saúde (BVS) e site do Ministério da Saúde. Para a busca dos artigos foi utilizado o operador booleano AND, com os seguintes Descritores em Ciências da Saúde (DeCS): "Gravidez", "Assistência pré-natal", "Gestação de alto risco" e "Complicações na gravidez".

Os filtros utilizados para atender os critérios de inclusão foram definidos: artigos gratuitos, disponíveis na íntegra, com ano de publicação nos últimos cinco anos. Assim, excluíram-se, artigos com ano de publicação inferior, pagos, incompletos, sem resumo nas bases de dados e as duplicidades de artigos. Ao aplicar os DeCS na busca, foram encontrados 100 artigos, após aplicação dos critérios de inclusão e exclusão foram selecionados 10 artigos para compor a análise dos dados.

\section{RESULTADOS E DISCUSSÕES}

O estudo demostra uma análise nas intercorrências obstétricas que ocasionam o pré-natal de alto risco, em decorrência das pesquisas, observamos a prevalência das irregularidades que pode levar a prejudicar a gestante e o feto, dessa forma, é extremamente importante essa causa ser discutida e ressaltada em diversas maneiras para os profissionais de saúde e sociedade.

$\mathrm{Na}$ pesquisa realizada foram utilizados quatro artigos, selecionados por critérios de inclusão e exclusão e que respondiam a questão norteadora levantada inicialmente. A partir dos achados, uma tabela contendo artigos foi criada, para mostrar, de forma sucinta, os resultados em relação às intercorrências obstétricas que ocasionam o pré-natal de alto risco. 
Tabela 1 - Apresentação dos estudos selecionados após pesquisa (Autores, título do artigo, local de publicação e síntese dos resultados encontrados).

\begin{tabular}{|c|c|c|c|}
\hline Autores & Título do artigo & $\begin{array}{l}\text { Local de } \\
\text { publicação }\end{array}$ & $\begin{array}{l}\text { Síntese dos resultados } \\
\text { encontrados }\end{array}$ \\
\hline $\begin{array}{l}\text { SAMPAIO, Aline } \\
\text { Fernanda Silva; } \\
\text { ROCHA, Maria } \\
\text { José Francalino } \\
\text { da; LEAL, } \\
\text { Elaine Azevedo } \\
\text { Soares, } 2018\end{array}$ & $\begin{array}{l}\text { Gestação de alto risco: } \\
\text { perfil r clínico- } \\
\text { epidemiológico das } \\
\text { gestantes atendidas } \\
\text { no serviço de pré-natal } \\
\text { da maternidade } \\
\text { pública de Rio Branco }\end{array}$ & $\begin{array}{l}\text { Revista } \\
\text { Brasileira } \\
\text { Saúde } \\
\text { Materno } \\
\text { Infantil }\end{array}$ & $\begin{array}{l}\text { Os autores falam a respeito das } \\
\text { alterações fisiológicas da gestação, e } \\
\text { que isso pode desenvolver condições } \\
\text { clínicas ou obstétricas desfavoráveis. } \\
\text { Evidenciam também que o maior } \\
\text { fator clínico de risco pré-gestacional } \\
\text { encontrado é a obesidade. }\end{array}$ \\
\hline $\begin{array}{l}\text { GADELHA, } \\
\text { Ivyna Pires et } \\
\text { al.: } 2020\end{array}$ & $\begin{array}{l}\text { Qualidade de vida das } \\
\text { mulheres com } \\
\text { gravidez de alto risco } \\
\text { durante o cuidado pré- } \\
\text { natal }\end{array}$ & $\begin{array}{l}\text { Revista } \\
\text { Brasileira } \\
\text { Enfermagem }\end{array}$ & $\begin{array}{l}\text { Neste estudo, os autores buscaram } \\
\text { analisar a qualidade de vida de } \\
\text { gestantes de alto risco, e puderam } \\
\text { notar que as mesmas têm maior } \\
\text { medo e ansiedade devidos as } \\
\text { condições de riscos gestacionais. }\end{array}$ \\
\hline $\begin{array}{l}\text { SANTOS, } \\
\text { Heliane } \\
\text { Fernandes } \\
\text { Lourenço; } \\
\text { ARAUJO, Marlei } \\
\text { Monteiro, 2016. } \\
\end{array}$ & $\begin{array}{l}\text { Políticas } \\
\text { humanização ao pré- } \\
\text { natal e parto: uma } \\
\text { revisão de literatura }\end{array}$ & $\begin{array}{l}\text { Revista } \\
\text { Científica } \\
\text { FacMais }\end{array}$ & $\begin{array}{l}\text { Os autores realizaram uma revisão } \\
\text { de literatura a respeito da Política de } \\
\text { Humanização ao Pré-natal, parto e } \\
\text { nascimento, que foi instituída com o } \\
\text { objetivo da redução das taxas de } \\
\text { mortalidade materna. }\end{array}$ \\
\hline $\begin{array}{l}\text { SOARES, } \\
\text { Agdaiane } \\
\text { Moura; } \\
\text { BORGES, } \\
\text { Luana Márcia } \\
\text { de Sousa; } \\
\text { BORGES, } \\
\text { Márcia Maria } \\
\text { Sousa: } 2016\end{array}$ & $\begin{array}{l}\text { Principais } \\
\text { intercorrências } \\
\text { complicações } \\
\text { obstétricas no período } \\
\text { da gestação e } \\
\text { puerpério }\end{array}$ & $\begin{array}{l}\text { Biblioteca } \\
\text { Atualiza }\end{array}$ & $\begin{array}{l}\text { O estudo mostra que a maior causa } \\
\text { de mortalidade materna no Brasil é } \\
\text { decorrente das síndromes } \\
\text { hipertensivas, também conhecidas } \\
\text { como doença hipertensiva específica } \\
\text { da gravidez, que acomete } 19 \% \text { das } \\
\text { gestantes no Brasil. }\end{array}$ \\
\hline
\end{tabular}

Fonte: dados da pesquisa, 2021.

A gravidez é um fenômeno fisiológico que ocorre devido à fecundação do óvulo com o espermatozoide, marcado pelo desenvolvimento e nascimento de um novo ser. Durante a gestação, a mulher sofre alterações fisiológicas que envolvem mudanças físicas, emocional e social, nesse momento a gestante pode desenvolver condições clínicas ou obstétricas desfavoráveis, o que irá desencadear uma gestação de alto risco, nesses casos, a mulher necessita de um cuidado mais ampliado (SAMPAIO; ROCHA; LEAL, 2018). Ela vai ser analisada pelo profissional durante o pré-natal quanto ao local: gravidez tópica ou ectópica; quanto à quantidade de embriões: única ou gemelar; e quanto ao risco: risco habitual ou alto risco (BRASIL, 2021). 
Estudo publicado por Gadelha et al. (2020) evidencia que as gestantes têm como principais pontos desfavoráveis que interferem na gravidez as condições financeiras, físicas, emocionais, de saúde e do sono, entretanto, outras condições, como as características clínicas e obstétricas da paciente como, por exemplo, idade e número de filhos interferem nos aspectos psicossociais e, consequentemente, alteram a qualidade de vida dessa mulher. Sendo assim, é importante realizar, nas consultas de pré-natal, uma conversa ampliada e um olhar holístico e individualizado a cada paciente, a fim de traçar estratégias em conjunto com essa mulher, para melhorar a sua qualidade de vida.

O Ministério da Saúde, com o objetivo de reduzir as taxas de mortalidade materna, neonatal e perinatal decidiu criar medidas para melhorar a assistência de saúde das gestantes e puérperas, e instituiu, em 2000, o Programa de Humanização de Pré-natal e Nascimento (PHPN), a fim de garantir o direito, a qualidade e o atendimento digno durante a gestação o parto e o puerpério, e que seja efetuado de forma segura e humanizada. Logo em seguida, no ano de 2003, o Ministério da Saúde criou outra portaria de $n^{\circ}$ 653/GM, a qual estabelece que o óbito materno seja um evento de notificação compulsória incluindo a causa da morte ou as medidas utilizadas para evitar tal acontecimento (SANTOS; ARAÚJO, 2016).

Outra estratégia do Governo Federal foi lançada em 2011, e garante à mulher o direito de realizar o planejamento familiar e uma atenção humanizada na gravidez, parto e puerpério. A Rede Cegonha tem como objetivo garantir os direitos sexuais de mulheres e homens, diminuir as taxas de mortalidade infantil, como também assegurar que as mulheres tenham qualidade de vida e bem-estar durante todo período gestacional, no parto e no puerpério e ainda assegurar o desenvolvimento saudável da criança até os dois anos de vida. A Rede Cegonha apresenta como um instrumento fundamental nas consultas de pré-natal a caderneta da gestante, onde devem ser registradas todas as informações importantes de procedimentos realizados durante a gestação, de modo a auxiliar o profissional na continuidade do atendimento (BRASIL, 2017).

É importante que o pré-natal seja iniciado nas primeiras semanas da gestação, de acordo com o Ministério da Saúde, preferencialmente antes da $12^{\circ}$ semana de gestação ou até mesmo quando a mulher apresentar a suspeita da 
gravidez já procurar uma unidade de saúde, juntamente com o seu parceiro, para que receba um melhor acompanhamento (BRASIL, 2021).

As consultas de pré-natal funcionam como uma troca de informações e experiências entre enfermeiros e médicos e as mulheres, que irão auxiliar a compreender melhor esse momento de mudanças. É no pré-natal o momento adequado para prevenir ou detectar fatores que venham a ocasionar riscos através da proteção vacinal, solicitação de exames de rotina e de consultas médicas (BRASIL, 2016).

O objetivo das consultas de pré-natal é garantir que a gravidez prossiga sem impacto para a vida materna. Inclui consultas, tratamentos psicossociais, atividades preventivas e educativas e, assim, assegurar o desenvolvimento da gestação e o nascimento de uma criança saudável (BRASIL, 2012).

A Organização Mundial da Saúde (OMS) afirma que o número adequado de consultas é igual ou superior a seis consultas durante toda a gestação, entretanto, considerando a importância de iniciar as consultas de pré-natal desde o início da gestação, segundo o Ministério da Saúde, a gestante deve realizar uma consulta mensal até a vigésima oitava semana, quinzenalmente até a trigésima sexta semana e semanais no termo, então, entende-se que se a mulher é acompanhada no prénatal desde o início da gestação, onde serão realizadas 13 consultas antes do parto (BRASIL, 2012).

Algumas vantagens do pré-natal é identificar doenças existentes antes mesmo da gestação, a avaliação placentária e qualquer alteração existente, más formações fetais, detectar a pré-eclâmpsia, que é uma das maiores causas de mortalidade materno-infantil, preparar a mulher para a maternidade e, com isso, reduzir as taxas de morbimortalidade (BRASIL, 2016).

$\mathrm{Na}$ gravidez de alto risco, nota-se que existe um maior índice de negação e aceitação por parte da gestante, o sentimento de incapacidade e de culpa podem aumentar os anseios dessas mulheres e, quando somados a causa da intercorrência gestacional, é possível observar uma considerável diminuição da qualidade de vida e bem-estar da puérpera (BRASIL, 2012).

Gadelha et al. (2020) também falam sobre essas alterações psicossociais presenciadas na gestação, e afirmam que, além das mudanças sociais e 
emocionais, o medo e a ansiedade também podem estar presentes para a maioria das mulheres, principalmente aquelas com fatores de risco associados, e afirmam que essas mudanças emocionais podem ser responsáveis por impactar a qualidade de vida das gestantes, então, é importante que, ao ser percebido esse sofrimento emocional, a mulher receba acompanhamento de um profissional qualificado.

$\mathrm{Na}$ assistência ao pré-natal, são possíveis que sejam identificados fatores que possam ocasionar riscos à gestação; para isso, é importante que o profissional realize os registros no cartão da gestante e esteja atento a qualquer alteração observada e que possa evoluir para um prognóstico negativo, pois é fato que uma gravidez saudável, de risco habitual, possa evoluir repentinamente para uma gravidez de alto risco ainda na gestação ou até mesmo no momento do parto (BRASIL, 2012).

Os fatores de riscos preexistentes são caracterizados quanto às condições sociodemográficas e individuais que incluem idade, escolaridade, hábitos de vida, renda, como também concepções anteriores e condições clínicas já apresentadas antes da gravidez, como hipertensão arterial, diabetes, obesidade, doenças infecciosas, autoimunes, cardíacas, pulmonares, renais, neurológicas ou outras que coloquem em risco a vida da mãe ou do feto. Os fatores adquiridos durante a gestação, que evoluem como risco gestacional, são doenças obstétricas diagnosticadas na gravidez atual como, por exemplo, hemorragias, pré-eclâmpsia ou eclâmpsia, diabetes gestacional, quantidade de fetos e de líquido amniótico, infecção por doenças infectocontagiosas, exposição a agentes teratogênicos ou óbito (BRASIL, 2012).

Conforme citado acima, é possível notar que o maior fator clínico de risco prégestacional encontrado é a obesidade, e isso se deve à diminuição da desnutrição e o aumento da obesidade na população brasileira, diz ainda que um ponto muito importante que deve ser abordado na assistência ao pré-natal é a avaliação nutricional das gestantes (SAMPAIO; ROCHA; LEAL, 2018).

A principal intercorrência gestacional, e maior causa de mortalidade materna no Brasil, é decorrente das síndromes hipertensivas, também conhecidas como doença hipertensiva específica da gravidez, que acomete 19\% das gestantes no Brasil e pode ocorrer nos períodos pré-termo, termo e pós-termo imediato, em casos 
mais graves evolui para a pré-eclâmpsia, eclâmpsia ou síndrome de HELLP. Outra intercorrência de grande percentual é a oligodramnia, caracterizada pela diminuição do líquido amniótico é possível perceber alteração durante o pré-natal através da palpação do abdome como também na avaliação da altura uterina (SOARES; BORGES; BORGES, 2016).

Sampaio, Rocha e Leal (2018) afirmam que nas consultas de pré-natal não é possível detectar as complicações que serão enfrentadas ao longo da gestação ou no momento do parto, isso pode ser notado através do número de mortes maternoinfantis que ainda são altos, porém, a identificação imediata dos fatores de riscos auxilia no tratamento e acompanhamento adequado, podendo resultar na continuidade da gestação e nascimento saudável da criança.

Soares, Borges e Borges (2016) falam sobre as principais complicações ocasionadas devido às intercorrências obstétricas, as mais comuns foram hemorragias no pós-parto e trabalho de parto prematuro, seguidos de crescimento intrauterino restrito, infecções do trato urinário e sofrimento fetal. Diante desses fatores, é imprescindível a realização de um tratamento adequado para as intercorrências detectadas podendo, então, assegurar à mulher uma menor probabilidade de complicações durante a gestação ou parto e, consequentemente, a diminuição das taxas de morte materna.

\section{CONCLUSÃO}

Diante dos estudos e documentos ministeriais que evidenciam a importância do pré-natal, como também a evolução das políticas de assistência às mulheres, vêse uma melhoria significativa nas taxas de mortalidade materna ao longo das últimas décadas após a implementação de estratégias baseadas na prevenção e vigilância de agravos. Apesar de toda mudança e esclarecimento a respeito do acompanhamento gestacional, ainda se consideram elevados os números de morbimortalidades materno-infantil. 
Portanto, deve-se haver uma investigação intersetorial mais aprofundada entre as equipes multiprofissionais de Atenção Básica e seguimentos especializados que atendem as gestantes, como também adesão, manutenção e melhorais das políticas já implantadas, buscando soluções para lacunas ainda existentes. Cabe ainda aos profissionais da saúde que trabalham na assistência primária a realização de um planejamento familiar de qualidade para que a mulher inicie precocemente a assistência pré-natal, busquem de forma ativa as gestantes que abandonaram as consultas, pratiquem uma atenção à saúde técnica igualmente respeitosa à gestante, de modo que ela esclareça suas dúvidas, estabeleça confiança nos profissionais e afirme seu compromisso de responsabilidade e amor com a nova vida que virá.

\section{REFERÊNCIAS BIBLIOGRÁFICAS}

BRASIL. Ministério da Saúde. Biblioteca Virtual em Saúde. Importância do pré-natal: 2016. Disponível em: https://bvsms.saude.gov.br/dicas-em-saude/2198-importancia-do-pre-natal.

BRASIL. Ministério da Saúde. Caderno de atenção básica- 32. Brasília: 2012. Disponível em: http://bvsms.saude.gov.br/bvs/publicacoes/cadernos_atencao_basica_32_prenatal.pdf.

BRASIL. Ministério da Saúde. Gravidez: o que é, tipos, sintomas complicações e prevenções. Brasília: 2021. Disponível em: https://antigo.saude.gov.br/saude-de-az/gravidez\#: :text=A\%20gravidez\%20\%C3\%A9\%20um\%20evento,e\%20para\%20toda\%20a\%20 fam\%C3\%ADlia.

BRASIL. Ministério da Saúde. Manual técnico de gestação de alto risco. Brasília: 2012. Disponível em: http://bvsms.saude.gov.br/bvs/publicacoes/manual_tecnico_gestacao_alto_risco.pdf.

BRASIL. Ministério da saúde. Rede Cegonha. Brasília: 2017. Disponível em: https://www.gov.br/saude/pt-br/acesso-a-informacao/acoes-e-programas/redecegonha\#: : :text=Sistema\%20log\%C3\%ADstico\%20(transporte\%20sanit\%C3\%A1rio\%20e,dois\% 20primeiros\%20anos\%20de\%20vida.

BRASILIA. Ministério da Saúde. Gabinete do Ministro. Portaria n 569: 2000. Disponível em: http://bvsms.saude.gov.br/bvs/saudelegis/gm/2000/prt0569_01_06_2000_rep.html.

GADELHA, Ivyna Pires et al. Qualidade de vida das mulheres com gravidez de alto risco durante - cuidado pré-natal. Rev. Bras. Enferm. 73 (suppl 5) •2020. Disponível em: https://doi.org/10.1590/0034-7167-2019-0595.

Mendes KDS, Silveira RCCP, Galvão CM. Revisão integrativa: método de pesquisa para a incorporação de evidências na saúde e na enfermagem. Texto Contexto Enferm. 2008;17(4): 758-64. 
SAMPAIO, Aline Fernanda Silva; ROCHA, Maria José Francalino da; LEAL, Elaine Azevedo Soares. Gestação de alto risco: perfil clínico-epidemiológico das gestantes atendidas no serviço de pré-natal da maternidade pública de Rio Branco, Acre. Rev. Bras. Saúde Mater. Infant. 18 (3). Jul-sep 2018. Disponível em: https://doi.org/10.1590/1806-93042018000300007.

SANTOS, Heliane Fernandes Lourenço; ARAUJO, Marlei Monteiro. Políticas de humanização ao pré-natal e parto: uma revisão de literatura. Revista Científica FacMais, Volume. VI, Número 2. Ano 2016/10 Semestre. ISSN 2238-8427. Disponível em: https://revistacientifica.facmais.com.br/wp-content/uploads/2016/07/Artigo-6-

POL\%C3\%8DTICAS-DE-HUMANIZA\%C3\%87\%C3\%83O-AO-PR\%C3\%89-NATAL-EPARTO.pdf.

SOARES, Agdaiane Moura; BORGES, Luana Márcia de Sousa; BORGES, Márcia Maria Sousa. Principais intercorrências e complicações obstétricas no período da gestação e puerpério. Trabalho de Conclusão de Curso de Especialização em Obstetrícia. Salvador - BA: Atualiza, 2016. Disponível em: http://bibliotecaatualiza.com.br/arquivotcc/EOB/EOB12/SOARESagdaiane-BORGES-luana-BORGES-marcia.pdf. 